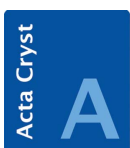

FOUNDATIONS

ADVANCES

ISSN 2053-2733

Received 7 June 2019

Accepted 28 June 2019

Edited by A. Altomare, Institute of

Crystallography - CNR, Bari, Italy

Keywords: symmetries; metric tensor; lattice; point group

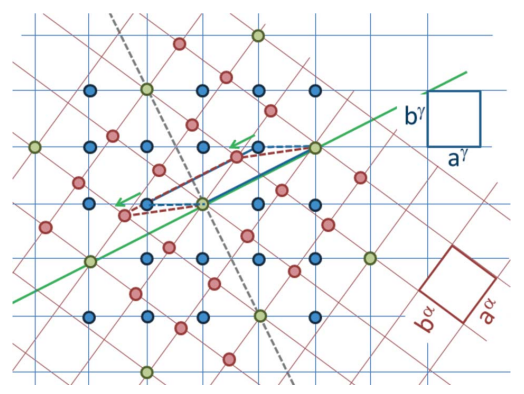

C 2019 International Union of Crystallography

\section{The transformation matrices (distortion, orientation, correspondence), their continuous forms and their variants. Corrigenda}

\author{
Cyril Cayron*
}

Laboratory of ThermoMechanical Metallurgy (LMTM), PX Group Chair, Ecole Polytechnique Fédérale de Lausanne (EPFL), Rue de la Maladière, 71b, Neuchâtel, 2000, Switzerland. *Correspondence e-mail: cyril.cayron@epfl.ch

Appendices B4 and B5 of Cayron [Acta Cryst. (2019), A75, 411-437] contain equations involving the point group and the metric tensor in which the equality symbol should be substituted by the inclusion symbol.

In Appendices B4 and B5 of Cayron (2019), we confused different notations related to the point groups, Laue groups and lattice groups. We denote the point group by $\mathbb{G}$ and the metric tensor by $\mathcal{M}$. As the symmetries preserve the norms and the angles, it is correct to write that $g^{\mathrm{t}} \mathcal{M g}=\mathcal{M}$ for any symmetry matrix $g$. However, the reciprocal is not always true. For example, a non-centrosymmetric crystal has its metric preserved by the inversion, but the inversion is not an element of $\mathbb{G}$. Consequently, equation (53) is incorrect and should be substituted by

$$
\mathbb{G} \subseteq\left\{g \in \pm \operatorname{SL}(N, \mathbb{Z}), g^{\mathrm{t}} \mathcal{M} g=\mathcal{M}\right\}
$$

Equation (55) should also be modified by replacing the first equals sign in each line by ' $\subseteq$ ', and equation (56) no longer holds. The confusion is inexcusable, but fortunately it does not affect all the other results presented in the paper.

Note: there is also a typographical error in Section 7.7. The lattice parameter $a^{\gamma}$ should not appear in the value of the shear amplitude: $s=[(1 / r)-r]$.

\section{References}

Cayron, C. (2019). Acta Cryst. A75, 411-437. 